\title{
A CONFORMAL SELF-MAP WHICH FIXES THREE POINTS IS THE IDENTITY
}

\author{
ERNST PESCHL and MATTI LEHTINEN
}

Recently I. Lieb raised the question of fixed points of conformal self-maps of plane domains with arbitrary connectivity. He conjectured the following

Theorem. Let $A$ be a plane domain and $f: A \rightarrow A$ a conformal map. If $f$ has three fixed points, then $f$ is the identity map.

In fact, this theorem is an immediate consequence of a result by B. Maskit [2], which states that one may associate with $A$ another domain $A^{\prime}$, conformally equivalent to $A$, such that all conformal self-maps of $A^{\prime}$ are Möbius transformations. Maskit's proof is rather elaborate, as it depends heavily on the structure of the group of conformal self-maps of $A$. In this note we attempt to give a straightforward and relatively short proof of the theorem.

We first remark that there exist domains of arbitrarily high connectivity allowing conformal self-maps with two fixed points, other than the identity. As an example one may consider any domain $A$ obtained from the plane when the origin and an arbitrary closed set of points on the unit circle, symmetric with respect to the real axis and not containing the points -1 and 1 , are deleted. Then $f, f(z)=1 / z$, maps $A$ onto itself and fixes -1 and 1 .

If $A$ is simply or doubly connected, consideration of all possible standard domains and their conformal self-maps [3, pp. 226-236] shows that the theorem is true in this case. In the sequel, therefore, we may assume that $A$ is at least triply connected. The unit disc $D$ can then be taken to be the universal covering surface of $A$, and the cover transformation group $G$ of $D$ relative to $A$ is a non-elementary properly discontinuous group of Möbius transformations.

In what follows we let $f$ be a fixed conformal self-map of $A$ and assume that $f\left(w_{0}\right)=w_{0}$ for some $w_{0}$ in $A$. Denote the projection map of $D$ onto $A$ by $h$; there is no loss of generality in assuming $h(0)=w_{0}$. Also, we may fix a lifting $\tilde{f}: D \rightarrow D$ of $f$ such that $\tilde{f}(0)=0$. Denote the $n$-th iterate of $\tilde{f}$ by $\tilde{f}^{n}$. We then have

Lemma. There exists a natural number $p$ such that $\tilde{f}^{p}=\mathrm{id}$.

Proof. Evidently $\tilde{f}(z)=\exp (i a) z$ for some real $a$. Choose a $g_{0}$ in $G, g_{0} \neq$ id. Then $g_{n}=\tilde{f}^{n} \circ g_{0} \circ\left(\tilde{f}^{n}\right)^{-1}$ is in $G$. If the number of distinct $g_{n}$ 's is infinite, a sequence $\left(g_{n_{j}}\right)$ converges to a conformal $g$. The discontinuity of $G$ makes this impossible. 
Consequently $g_{n}=g_{n+p}$ for some $n$ and $p \geqq 1$. In particular, then, $\exp (i a(n+p)) g_{0}(0)=$ $\exp ($ ian $) g_{0}(0)$. But 0 is not a fixed point of $g_{0}$, and we may cancel by $\exp ($ ian $) g_{0}(0)$ to obtain the assertion of the Lemma.

To proceed in the proof of the Theorem, let us denote by $F$ the set of points $z$ in $D$, such that $h(z)$ is a fixed point of $f$, but $h(z) \neq w_{0}$. Assume $F$ to be non-empty. The set $F$ has a positive Euclidean distance $r$ from 0 . Choose $z_{1}$ in $F$ such that $\left|z_{1}\right|=r$, and let $p$ be the smallest natural number such that $\tilde{f}^{p}=$ id. Set $z_{k}=\tilde{f}\left(z_{k-1}\right)$, $k=2, \ldots, p$, and join every $z_{k}$ to 0 by the line segment $\tilde{\alpha}_{k}$. Then $\alpha_{k}=h\left(\tilde{\alpha}_{k}\right)$ is a path joining $w_{1}=h\left(z_{1}\right)$ to $w_{0}$. Moreover, $\alpha_{k}$ is a Jordan arc. For assume $h\left(t_{1}\right)=h\left(t_{2}\right)$, where $t_{1}, t_{2} \in \tilde{\alpha}_{k}$, and $\left|t_{1}\right|<\left|t_{2}\right|$. Then $w_{0}$ and $w_{1}$ are joined by $h(\beta)$, where $\beta$ consists of the segment $\left(0, t_{1}\right)$ and a circular arc congruent modulo $G$ with the segment $\left(t_{2}, z_{k}\right)$. The hyperbolic length of $\beta$ is strictly smaller than that of $\tilde{\alpha}_{k}$, but the Euclidean distance of 0 from the other end-point of $\beta$ is at least $r$. This is in contradiction with the fact that rays issuing from the origin are geodesics in the hyperbolic metric.

A similar argument shows that for $k \neq j, \alpha_{k}$ and $\alpha_{j}$ cannot meet except at $w_{0}$ and $w_{1}$. Assume $h\left(t_{k}\right)=h\left(t_{j}\right)$ with $t_{k} \in \tilde{\alpha}_{k}, t_{j} \in \tilde{\alpha}_{j}$. Because $h\left(t_{k}\right)$ is not a fixed point of $f$, we may suppose $\left|t_{k}\right|<\left|t_{j}\right|$. It follows that $w_{0}$ and $w_{1}$ are joined by $h(\gamma)$, where $\gamma$ is composed of the segment $\left(0, t_{k}\right)$ and a circular arc congruent modulo $G$ with the segment $\left(t_{j}, z_{j}\right)$. Again, the hyperbolic length of $\gamma$ is strictly less than that of $\tilde{\alpha}_{k}$, while the Euclidean distance of 0 from the other end-point of $\gamma$ is at least $r$.

To complete the proof, assume $w_{2}$ is a fixed point of $f$, distinct from $w_{0}$ and $w_{1}$. One may join $w_{2}$, which does not lie on any $\alpha_{k}$, to $w_{0}$ by an arc $\alpha$ which does not meet any $\alpha_{k}$. Then $\alpha$ lies in the Jordan domain $B$ (of the extended plane) bounded by, say, $\alpha_{k}$ and $\alpha_{j}$, where $\alpha_{k}$ and $\alpha_{j}$ are adjacent segments. Consider the lifting $\tilde{\alpha}$ of $\alpha$, with initial point 0 . Since $h$ is a local homeomorphism at 0 , we see that $f(\alpha)$ must emerge from $w_{0}$ into the complement of $B$. Then $f(\alpha)$ must have a point in common either with $\alpha_{k}$ or $\alpha_{j}$. But this is impossible, since $f$ carries the set of arcs $\alpha_{k}$ onto itself [4].

Remark. Another proof for the Theorem above has been found by K. Leschinger (Bonn) [1].

\section{References}

[1] LesChinger, K.: Über Fixpunkte holomorpher Automorphismen. - Manuscripta Math. 25, 1978, 391-396.

[2] Maskit, B.: The conformal group of a plane domain. - Amer. J. Math. 90, 1968, 718-722.

[3] Peschl, E.: Funktionentheorie I. - Bibliographisches Institut, Mannheim, 1967.

[4] Peschl, E.: Über das Fixpunktverhalten von Automorphismen beliebiger Bereiche. - Preprint no. 173 (Januar 1978) Universität Bonn (Sonderforschungsbereich 72), 1-10.

Universität Bonn

Mathematisches Institut

D-5300 Bonn

BRD
University of Helsinki

Department of Mathematics

SF-00100 Helsinki 10

Finland

Received 24 April 1978 